\title{
Classification of Unconstrained Respiratory States Utilising Multidimensional Probability Distribution Based on Respiratory Frequency Information at Each Time Step
}

\author{
Misaki KOHAMA*, Yuri HAMADA*, Takashi KABURAGI** and Yosuke KURIHARA* \\ * Aoyama Gakuin University, 5-10-1 Fuchinobe, Chuo-ku, Sagamihara-shi, Kanagawa 252-5258, Japan \\ ** International Christian University, 3-10-2, Osawa, Mitaka-shi, Tokyo 181-8585, Japan
}

\begin{abstract}
In this study, an unconstrained respiratory state classification system is proposed at each time step to detect the symptoms of sleep apnoea. An air mattress-type pressure sensor was developed to unconstrainedly measure the respiration signal during sleep. Based on the measurements, an algorithm that can classify respiratory states by applying a multidimensional probability distribution is proposed. Two types of validity experiments were conducted. In the first experiment, it was verified whether the respiration signal could be accurately measured by the developed pressure sensor. The results showed an average absolute error of $0.3 \mathrm{br} / \mathrm{min}$. In the second experiment, the robustness of the classification accuracy to variations in the physical characteristics of the participants and recumbent positions was verified. The results showed an average F-value of 0.83 when extreme value distribution was applied. The classification accuracy of the proposed method outperformed the simple threshold method and the authors' previous work.
\end{abstract}

Keywords: Pressure sensor, Respiration signal, Sleep apnoea

\section{INTRODUCTION}

Obstructive sleep apnoea is a common sleep disorder. Although 930 million adults are estimated to have sleep apnoea worldwide [1], the diagnosis rate is low at approximately $15 \%$. Hence, the duration of respiratory arrests during sleep should be monitored regularly in a home environment, because this objective evaluation educates potential patients of their symptoms and encourages them to take appropriate countermeasures at a medical institution. Wearable sensors have been developed for daily respiration monitoring [2-5]; however, such sensors can be a burden to the patients because they have to be worn. Therefore, unconstrained methods that can measure respiration, wherein no sensors need to be attached to the patient's body, have been proposed [6-12]. In such methods, the respiration signal is measured as a time-series waveform that synchronises with diaphragm and lung movements due to breathing, and the duration of the respiratory arrest is determined by reducing the amplitude of the waveform. However, since the signal is measured for the same time length as the sleep period, it is difficult for medical staff to detect the respiratory arrest period from all time-series waveforms by visual inspection. Therefore, a signal processing algorithm to automatically extract the duration of respiratory arrests from all time-series waveforms was proposed. The simplest approach for detecting respiratory arrests during sleep using an unconstrained method is to utilise a threshold for the time-series waveform obtained using the sensor. However, the amplitude of the signal varies with the physical characteristics of the patient, such as body weight and height, as well as their recumbent positions, and thus, the optimal threshold cannot be easily determined.

In [13], we proposed a signal processing algorithm that is robust to the characteristics of the patient that are based on their respiratory cycles, which is calculated by considering the difference in the autocorrelation. The time-series waveform was divided into fixed-length segments, and the algorithm classified the segments based on whether or not they included a respiratory arrest period. However, when a segment contained multiple respiratory arrest periods, the accuracy of the classification was reduced.

In this paper, to improve the accuracy of the respiratory arrest period classification, we propose a method that determines whether the patient is breathing or is experiencing a respiratory arrest at each discrete time step.

\section{PROPOSED METHOD}

In Section 2.1, we describe a system suitable for the unconstrained measurement of respiration signals. In Section 2.2, we explain the signal processing for the classification of respiratory states (i.e. breathing and respiratory arrest) using the respiration signals obtained by the proposed system. 


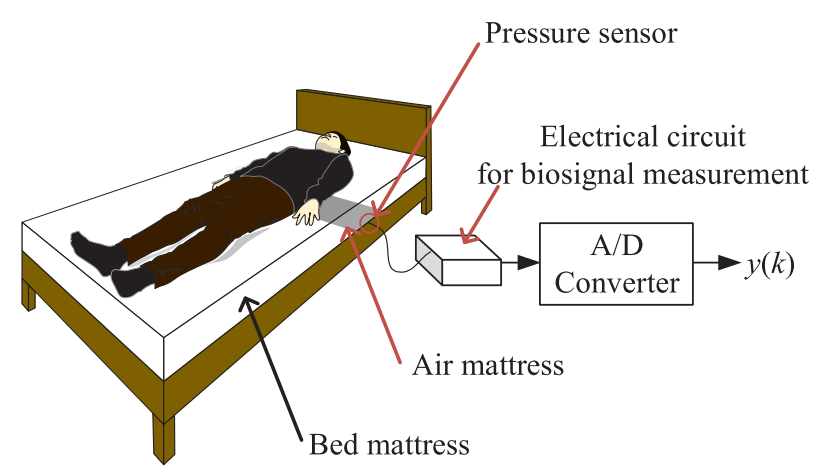

Figure 1: Unconstrained respiration measurement system

\subsection{Unconstrained Respiration Measurement System}

Figure 1 shows the unconstrained respiration signal measurement system.

The vibration of the diaphragm and lung movements due to respiration propagates to the air mattress via the bed mattress and changes the inner pressure of the air tube in the air mattress. One end of the air tube was sealed, and a pressure sensor was installed at the other end; therefore, the internal pressure of the tube that fluctuated in synchronisation with respiration was measured using the pressure sensor. The continuous signal output from the pressure sensor is passed through an $\mathrm{A} / \mathrm{D}$ converter to the computer as a discrete signal $y(k)$. Let $k$ be discrete time steps $(k=1,2,3 \ldots)$.

Since the size of the lungs is closely related to the physical characteristics of the patient, such as their weight and height, the respiration amplitude of the $y(k)$ for each patient is different. In addition, the contact area between the bed mattress and the chest varies depending on the person's recumbent position, which affects the vibration propagation efficiency and amplitude of the pressure sensor output. Thus, the measured signal $y(k)$ depends considerably on the characteristics of the patients as well as their recumbent positions.

\subsection{Signal Processing Flow to Classify Respiratory States}

Figure 2 shows an overview of the proposed signal processing algorithm for respiratory states classification based on the signals $y(k)$ measured using the unconstrained respiration measurement system. The algorithm consists of the preparation phase and the estimation phase.

In this study, to ensure that the variations in the physical characteristics of the participants and their recumbent positions did not affect the classification of respiratory states, frequency information was used. We assume that different respiratory states have different frequency information. In addition, the frequency information at each discrete time step is assumed to fluctuate probabilistically owing to individual differences and changes in frequency information. We utilised this probabilistic behaviour and classified the respiratory states using a probability distribution.

In the preparation phase, two types of respiration signals $y_{r}(k)$ and $y_{a}(k)$ were obtained using the system described in Section 2.1. $y_{r}(k)$ contains only the breathing period, and $y_{a}(k)$ contains only the respiratory arrest period. To obtain the frequency characteristics at each discrete time step $k$, the wavelet transform is applied to $\mathrm{y}_{r}(k)$ and $y_{a}(k)$ such that the wavelet coefficient matrices $W_{r}(k, f)$ and $W_{a}(k, f)$ with discrete time step $k$ and discrete frequency $f$ are obtained. The frequency band corresponding to the respiration of the participant was extracted from the wavelet coefficient matrix. The
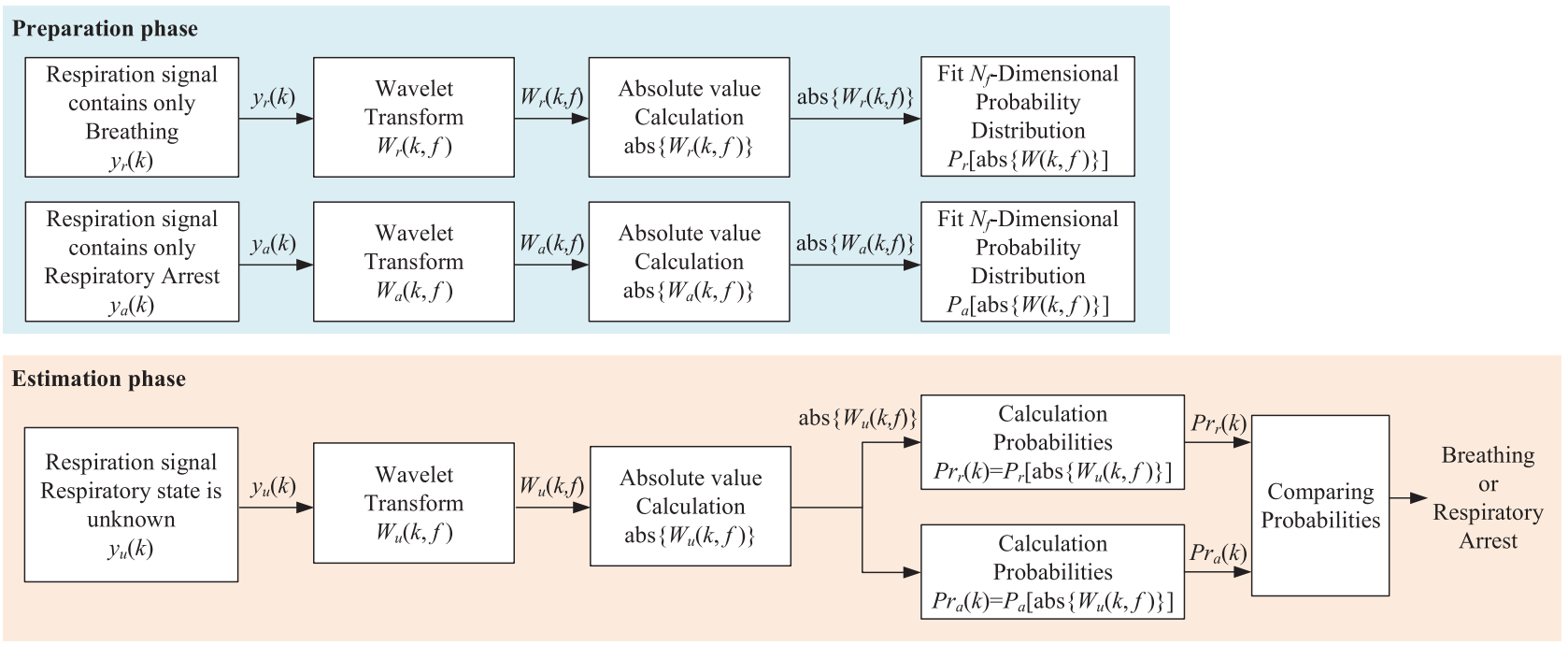

Figure 2: Proposed method 
general respiratory frequency band is $0.2-0.33 \mathrm{~Hz}$; however, considering individual differences such as tachypnoea, we set a wider respiratory unique frequency band of $0.15-0.45 \mathrm{~Hz}$. Let the $N_{f}$ be the number of discrete frequencies $f$ corresponding to the respiratory frequency band $0.15-0.45 \mathrm{~Hz}$. Thus, we assume that the absolute values of the wavelet coefficient matrices $\operatorname{abs}\left\{W_{r}(k, f)\right\}$ and $\operatorname{abs}\left\{W_{a}(k, f)\right\}$ are $N_{f}$-dimensional random variables. We constructed probability distributions for breathing $P_{r}[\operatorname{abs}\{W(k, f)\}]$ and for respiratory arrest $P_{a}[\operatorname{abs}\{W(k, f)\}]$ by fitting a $N_{f}$-dimensional probability distribution.

In the estimation phase, the absolute value of the wavelet coefficient matrix abs $\left\{W_{u}(k, f)\right\}$ is obtained via signal processing, which is realised in the preparation phase for signals $y_{u}(k)$ whose respiratory state is unknown. By calculating the probability of the $N_{f}$-dimensional random variables $\operatorname{abs}\left\{W_{u}(k, f)\right\}$ at each discrete time step $k$ for the two types of probability distributions $P_{r}[\operatorname{abs}\{W(k, f)\}]$ and $P_{a}[\operatorname{abs}\{W(k, f)\}]$ constructed in the preparation phase, we obtain the probability of breathing $\operatorname{Pr}_{r}(\mathrm{k})=P_{r}\left[\operatorname{abs}\left\{W_{u}(k, f)\right\}\right]$ and respiratory arrest $\operatorname{Pr}_{a}(\mathrm{k})=P_{a}\left[\operatorname{abs}\left\{W_{u}(k, f)\right\}\right]$. The respiratory states are classified by comparing probabilities $\operatorname{Pr}_{r}(k)$ and $\operatorname{Pr}_{a}(k)$. If the probability of respiratory arrest $\operatorname{Pr}_{a}(k)$ is larger than that of breathing $\operatorname{Pr}_{r}(k)$, the discrete time step $k$ is assumed to be a respiratory arrest period. In contrast, if the probability of breathing $\operatorname{Pr}_{r}(k)$ is larger than that of respiratory arrest $\operatorname{Pr}_{a}(k)$, the discrete time step $k$ is assumed to be a breathing period.

\section{VERIFICATION EXPERIMENT}

To validate the proposed method, we conducted a respiration signal measurement (RSM) experiment, in which accurate respiration signals were measured using the air mattress-type pressure sensor, and respiratory state classification (RSC) experiment, in which the proposed method was used to classify the respiratory period and respiratory arrest period for each time step $k$. All experiments were conducted after receiving an ethical review and approval from Aoyama Gakuin University (permission no. H21-004). Informed consent was obtained before initiation of the experiment.

\subsection{Experimental System}

Figure 3 shows the setup for both the experiments.

By placing the air mattress under the bed mattress, signals that account for the respiration, pulse, body movements, and noise of the participant lying on the bed

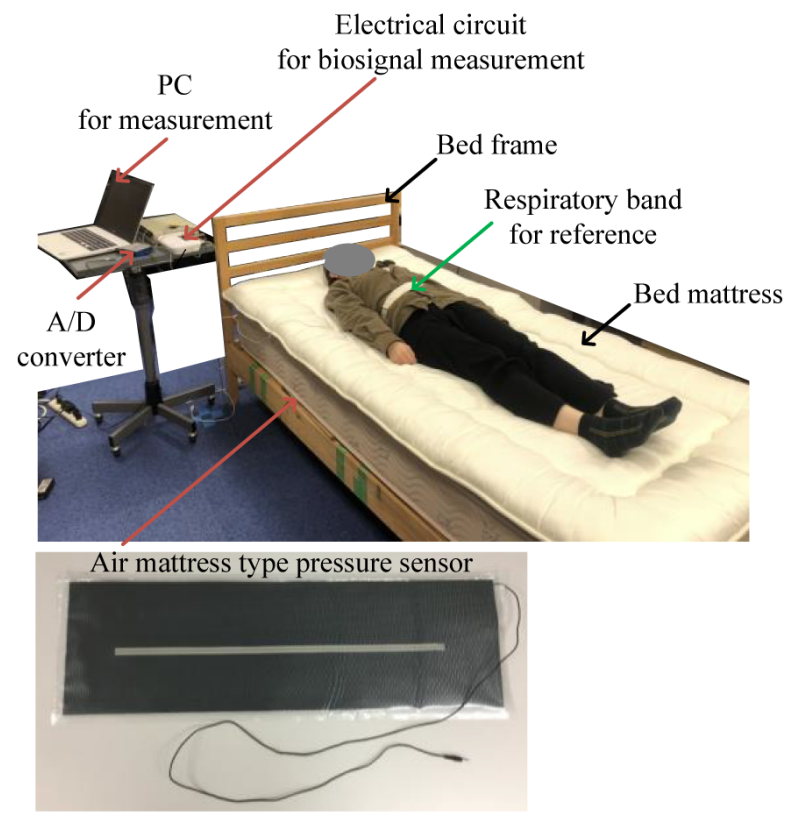

Figure 3: Experimental setup

could be measured. The air mattress was placed $65 \mathrm{~cm}$ from the head end of the frame such that the mattress was under the participant's chest. A bed mattress with a thickness of $20 \mathrm{~cm}$ was placed on top, and the participant lay down on it. The sampling frequency for the A/D conversion was set at $100 \mathrm{~Hz}$.

\subsection{Procedures and Participants}

\subsubsection{RSM Experiment}

In this RSM experiment, we verified whether the respiration signal could be accurately measured by an air mattress-type pressure sensor by comparing the respiration signal obtained from a respiratory band. The participant was asked to breathe while resting for $1 \mathrm{~min}$, and $y(k)$ of a one-minute time-series waveform was measured using the experimental system shown in Section 3.1. To obtain the respiration signal, a digital band-pass filter with cut-off frequencies ranging from 0.15 to $0.45 \mathrm{~Hz}$ is applied to the output signal from the pressure sensor $y(k)$. At this time, the participant was wearing a respiratory band around the abdomen to measure the respiration signal synchronised with the diaphragm movement as a reference, as shown in Figure 3. In this experiment, the participants were asked to lie down on the bed in four possible positions assumed during sleep: supine, right lateral, left lateral, and prone. We considered five participants with various heights and weights, and the details of the participants are listed in Table 2. Three male and two female participants with a height of $166.6 \pm 9.8 \mathrm{~cm}$ and a weight of $56.6 \pm 11.2 \mathrm{~kg}$ were included. The total 


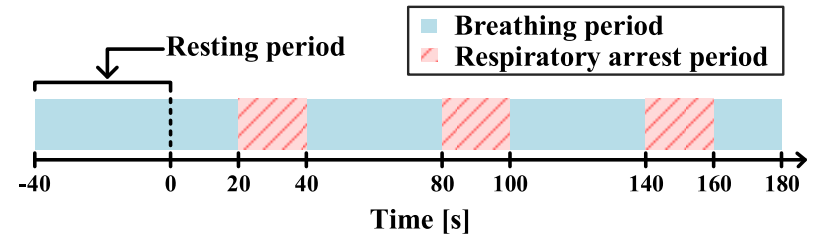

Figure 4: Experimental flow

number of $y(k)$ obtained was 20 (five participants $\times$ four positions).

\subsubsection{RSC Experiment}

In this RSC experiment, we verified the robustness of the classification accuracy of the proposed method to variations in the physical characteristics of the participants and their recumbent positions. The participants were asked to lie down on the bed in four possible sleeping positions during sleep: supine, right lateral, left lateral, and prone.

The experimental flow is shown in Figure 4.

First, the participant lay down on a bed mattress and was given a $40 \mathrm{~s}$ resting period before the data measurement. Then, simultaneously with the start of data measurement, a voice file created in MATLAB was executed. This voice file instructed the participant to stop breathing and reproduce the state of respiratory arrest. During the $180 \mathrm{~s}$ of data measurement, the participant was instructed to reproduce the respiratory arrest state for 20-40 s, 80-100 s, and $140-160 \mathrm{~s}$. The respiration signals are divided into two types of respiration signals: those containing solely breathing $y_{r}(k)$ and those containing solely respiratory arrest $y_{a}(k)$ with reference to the voice instruction. Thus, the number of $y_{r}(k)$ data points is 12000 and the number of $y_{a}(k)$ data points is 6000 from $180 \mathrm{~s}$ of data we obtained. The measurements were repeated for each sleeping position. In this verification experiment, we considered 10 participants with various heights and weights. Eight male and two female participants with a height of $170.0 \pm 8.2 \mathrm{~cm}$ and weight of $59.9 \pm 9.5 \mathrm{~kg}$. The total number of data obtained was 40 (ten participants $\times$ four positions)

The mother wavelet of the wavelet transform was Morlet and $N_{f}$ was set at 16 . In this experiment, as a 16-dimensional probability distribution, normal distribution, exponential distribution, extreme value distribution, gamma distribution, and lognormal distribution were applied to compare the classification accuracy. Here, the same type of probability distribution was applied to the probability distribution of breathing $P_{r}[\operatorname{abs}\{W(k, f)\}]$ and respiratory arrest $P_{a}[\operatorname{abs}\{W(k, f)\}]$. In the proposed method, the probability distributions were constructed using the absolute values of the wavelet coefficients
$\operatorname{abs}\left\{W_{r}(k, f)\right\}$ and $\operatorname{abs}\left\{W_{a}(k, f)\right\}$ for each discrete frequency at each discrete time, and the breathing or respiratory arrest was classified using the difference in the probability distributions between the breathing and respiratory arrest periods. Here, it was assumed that there is a difference in the probability of the abs $\left\{W_{r}(k, f)\right\}$ and $\operatorname{abs}\left\{W_{a}(k, f)\right\}$ for each frequency between the breathing and respiratory arrest periods. The study focused on the skewness of the probability distribution to find the optimal probability distribution for the proposed method for which the normal distribution, gamma distribution, extreme value distribution, and exponential distribution were selected. The normal distribution was chosen to construct a symmetric probability distribution. The gamma distribution was chosen such that a right-skewed probability distribution was modelled to characterise small values in $\operatorname{abs}\left\{W_{r}(k, f)\right\}$ and $\operatorname{abs}\left\{W_{a}(k, f)\right\}$ as high probabilities. In contrast, the extreme value distribution was chosen such that a left-skewed probability distribution was modelled to characterise large values in $\operatorname{abs}\{W r(k, f)\}$ and $\operatorname{abs}\left\{W_{a}(k, f)\right\}$ as high probabilities. In addition, as it is believed that $\operatorname{abs}\left\{W_{a}(k, f)\right\}$ tend to be smaller than abs $\left\{W_{r}(k, f)\right\}$, the exponential distribution was chosen as extremely right-skewed distribution which is a particular case of the gamma distribution. Furthermore, to evaluate the logarithmic characteristics of $\operatorname{abs}\left\{W_{r}(k, f)\right\}$ and $\operatorname{abs}\left\{W_{a}(k, f)\right\}$, the lognormal distribution was used as a probability distribution.

\subsection{Evaluation Method}

\subsubsection{Evaluation of Respiratory Rate for RSM Experiment}

As discussed in Section 3.1, the respiration signal of the abdomen was measured by the respiratory band simultaneously with the proposed system.

To verify whether the respiration signals could be measured accurately, FFT was performed on the respiration signals obtained from each of them, and the respiratory rate $(\mathrm{RR})$ values were calculated and compared. The performance was evaluated by calculating the absolute error of the RSM experiment.

\subsubsection{Evaluation of Respiratory States for RSC Experiment}

To determine the robustness to variations in the physical characteristics of the participants and their recumbent positions, the proposed system was evaluated via two cross-validation tests: leave-one-participant-out cross-validation and leave-one-recumbent-position-out cross-validation. The classification results are aggregated, as summarised in Table 1. 
Table 1: Aggregation method of classification

\begin{tabular}{|c|c|c|c|}
\hline \multicolumn{2}{|c|}{} & \multicolumn{2}{|c|}{ Classification Result } \\
\cline { 3 - 4 } & Respiratory Arrest & Breathing \\
\hline \multirow{2}{*}{ Reference } & $\begin{array}{c}\text { Respiratory } \\
\text { Arrest }\end{array}$ & $\begin{array}{c}\text { True Positive } \\
\text { (TP) }\end{array}$ & $\begin{array}{c}\text { False Negative } \\
\text { (FN) }\end{array}$ \\
\cline { 2 - 4 } & Breathing & $\begin{array}{c}\text { False Positive } \\
\text { (FP) }\end{array}$ & $\begin{array}{c}\text { True Negative } \\
\text { (TN) }\end{array}$ \\
\hline
\end{tabular}

TP, TN, FP, and FN represent "true positive" (respiratory arrest detected as respiratory arrest), "true negative" (breathing detected as breathing), "false positive" (breathing detected as respiratory arrest), and "false negative" (respiratory arrest detected as breathing), respectively. Furthermore, five indices were calculated based on TP, TN, FP, and FN, as follows:

$$
\begin{aligned}
& \text { Sensitivity }=\frac{\mathrm{TP}}{\mathrm{TP}+\mathrm{FN}} \\
& \text { Specificity }=\frac{\mathrm{TN}}{\mathrm{TN}+\mathrm{FP}} \\
& \mathrm{PPV}=\frac{\mathrm{TP}}{\mathrm{TP}+\mathrm{FP}} \\
& \mathrm{NPV}=\frac{\mathrm{TN}}{\mathrm{TN}+\mathrm{FN}} \\
& \mathrm{F}-\text { value }=\frac{2 \mathrm{TP}}{2 \mathrm{TP}+\mathrm{FN}+\mathrm{FP}}
\end{aligned}
$$

\section{EXPERIMENTAL RESULTS}

\subsection{Results of RSM Experiment}

\subsubsection{Typical Example of Respiration Signals}

Figure 5 shows the signals obtained from the RSM experiment, as described in Section 3.2.1.

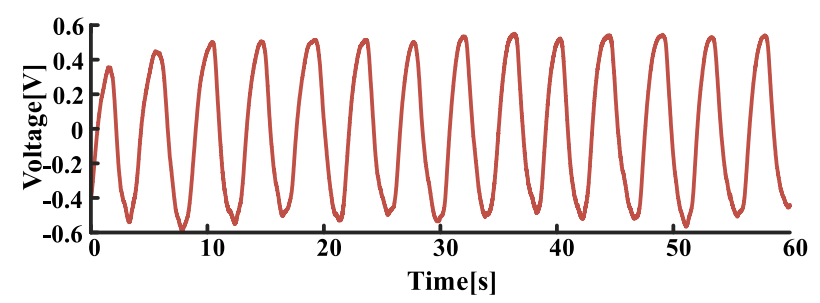

(a) Filtered output signal

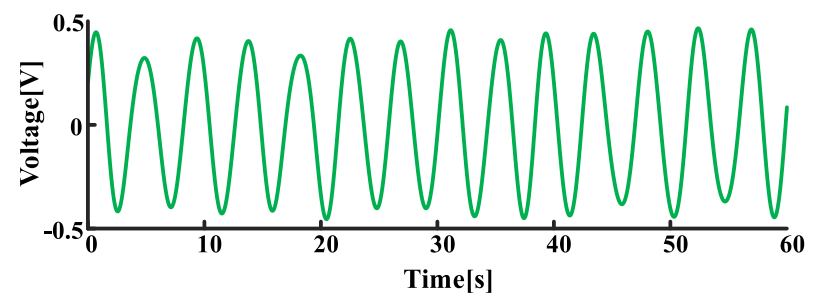

\begin{tabular}{|c|c|c|c|c|c|c|c|}
\hline \multirow{2}{*}{$\begin{array}{l}\text { Participant } \\
\text { ID }\end{array}$} & \multirow{2}{*}{ Gender } & \multirow{2}{*}{$\begin{array}{c}\text { Height } \\
(\mathrm{cm})\end{array}$} & \multirow{2}{*}{$\begin{array}{c}\text { Weight } \\
\text { (kg) }\end{array}$} & \multirow{2}{*}{$\begin{array}{l}\text { Recumbent } \\
\text { Position }\end{array}$} & \multicolumn{2}{|c|}{ RR (br/min) } & \multirow{2}{*}{$\begin{array}{l}\text { Absolute } \\
\text { Error }\end{array}$} \\
\hline & & & & & Estimated & Reference & \\
\hline \multirow{4}{*}{01} & \multirow{4}{*}{ M } & \multirow{4}{*}{164} & \multirow{4}{*}{55} & Supine & 14 & 14 & 0 \\
\hline & & & & Prone & 17 & 17 & 0 \\
\hline & & & & Right Lateral & 15 & 15 & 0 \\
\hline & & & & Left Lateral & 15 & 16 & 1 \\
\hline \multirow{4}{*}{02} & \multirow{4}{*}{$\mathrm{F}$} & \multirow{4}{*}{157} & \multirow{4}{*}{44} & Supine & 18 & 18 & 0 \\
\hline & & & & Prone & 14 & 14 & 0 \\
\hline & & & & Right Lateral & 18 & 17 & 0 \\
\hline & & & & Left Lateral & 17 & 17 & 0 \\
\hline \multirow{4}{*}{03} & \multirow{4}{*}{$\mathrm{F}$} & \multirow{4}{*}{156} & \multirow{4}{*}{45} & Supine & 12 & 13 & 1 \\
\hline & & & & Prone & 14 & 14 & 0 \\
\hline & & & & Right Lateral & $\frac{17}{13}$ & 13 & 0 \\
\hline & & & & Left Lateral & 15 & 15 & 0 \\
\hline \multirow{4}{*}{09} & \multirow{4}{*}{ M } & \multirow{4}{*}{180} & \multirow{4}{*}{69} & Supine & 20 & 20 & 0 \\
\hline & & & & Prone & 18 & 17 & 1 \\
\hline & & & & Right Lateral & 19 & 19 & 0 \\
\hline & & & & Left Lateral & 19 & 19 & 0 \\
\hline \multirow{4}{*}{10} & \multirow{4}{*}{ M } & \multirow{4}{*}{176} & \multirow{4}{*}{70} & Supine & 15 & 15 & 0 \\
\hline & & & & Prone & 19 & 16 & 3 \\
\hline & & & & Right Lateral & 14 & 14 & $\frac{0}{0}$ \\
\hline & & & & Left Lateral & 13 & 13 & 0 \\
\hline & & & & & & Average & 0.3 \\
\hline
\end{tabular}

(b) Respiratory band for Reference

Figure 5: Simultaneously measured respiration signals
Table 2: Comparison of the measurement results for the five participants

As shown in Figure 5, the filtered output signal from the air mattress-type pressure sensor was synchronised with the signal measured by the respiratory band for reference. This indicates that the proposed system can measure the respiration signal in an unconstrained manner.

\subsubsection{Comparison of Accuracy of Respiratory Rate by Reference}

Table 2 summarises the results of the comparison between the RR obtained by the proposed system and the reference RR.

The results of the comparison experiment with five participants showed an average absolute error of 0.3 .

\subsection{Results of RSC Experiment}

\subsubsection{Aggregation of Respiratory States Classification Result}

Figure 6 shows the signal obtained from the RSC experiment described in Section 3.2.2. Figure 6(a) shows the output $y(k)$ from the sensor. The red areas indicate the respiratory arrest period, $y_{a}(k)$, which were a result of the voice instructions. Figure 6(b) shows the wavelet scalogram obtained by the absolute value of the wavelet coefficient matrix $\operatorname{abs}\{W(k, f)\}$ of the waveform $y(k)$ in Figure 6(a). The three sets of vertical dashed lines indicate the periods during which the respiratory arrest state was reproduced. The natural respiratory frequency band of $0.15-0.45 \mathrm{~Hz}$ is shown as a horizontal dashed line on the wavelet scalogram.

During breathing, a large amplitude was observed in the respiratory frequency band; however, during respiratory arrest, this amplitude decreases.

Figure 7 shows the results of the RSC at each time step using the proposed method, when the wavelet coefficient matrix $W(k, f)$ in Figure $6(\mathrm{~b})$ is used as the test data. 


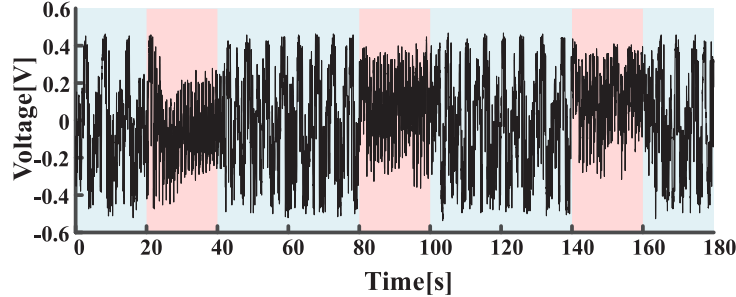

(a) Output signal

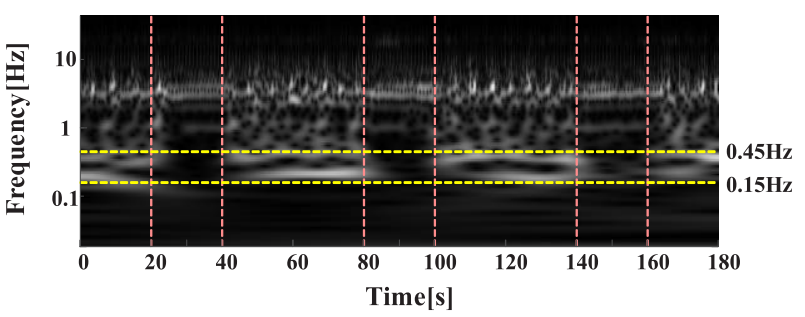

(b) Wavelet scalogram

Figure 6: Respiration signals measured by the proposed unconstrained respiration measurement system

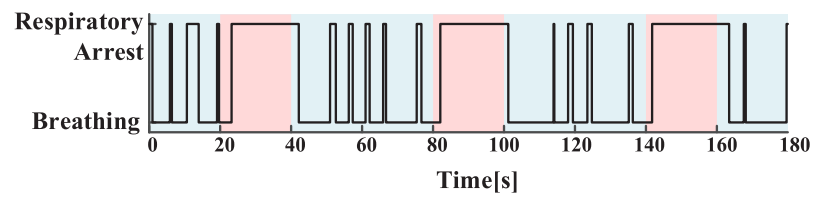

(a) Basic method

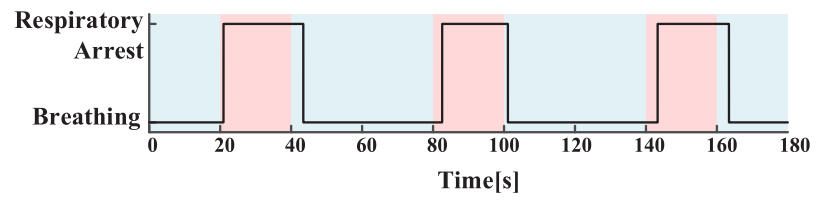

(b) Proposed method

Figure 7: Results of the classification respiratory states

Figure 7(a) shows the estimation result using the basic method with a threshold value optimised by the ROC curve. Figure 7(b) shows the classification results of the proposed method. The probability distribution in fitting the 16-dimensional probability distribution of the proposed method exhibits an extreme value distribution. The red areas indicate the respiratory arrest period that were a result of the voice instructions.

As shown in Figure 7, the proposed method could classify the respiratory states with high accuracy, whereas the basic method misclassified the breathing period for the respiratory arrest period at several time steps.

\subsubsection{Consideration of Probability Distributions}

In this section, we compare the results of the five indices for normal distribution, exponential distribution, extreme value distribution, gamma distribution, and lognormal distribution. Table 3 summarises the results of the evaluation indices for each probability distribution.
Table 3: Consideration of Probability Distributions

\begin{tabular}{c|c|c|c|c|c|c}
\hline \hline $\begin{array}{c}\text { Cross } \\
\text { validation }\end{array}$ & Distribution & Sen. & Spec. & PPV & NPV & F-value \\
\hline \hline \multirow{4}{*}{ Participant } & Normal & 0.76 & 0.95 & 0.90 & 0.89 & 0.82 \\
\cline { 2 - 7 } & Exponential & 0.61 & 0.99 & 0.96 & 0.84 & 0.75 \\
\cline { 2 - 7 } & Extreme Value & 0.84 & 0.90 & 0.82 & 0.92 & 0.83 \\
\cline { 2 - 7 } & Gamma & 0.65 & 0.98 & 0.95 & 0.85 & 0.77 \\
\cline { 2 - 7 } & Lognormal & 0.58 & 0.97 & 0.92 & 0.82 & 0.71 \\
\hline \multirow{4}{*}{$\begin{array}{c}\text { Recumbent } \\
\text { position }\end{array}$} & Normal & 0.77 & 0.95 & 0.89 & 0.89 & 0.82 \\
\cline { 2 - 7 } & Exponential & 0.62 & 0.99 & 0.96 & 0.84 & 0.75 \\
\cline { 2 - 7 } & Extreme Value & 0.84 & 0.90 & 0.81 & 0.83 & 0.83 \\
\cline { 2 - 7 } & Gamma & 0.66 & 0.98 & 0.95 & 0.85 & 0.78 \\
\cline { 2 - 7 } & Lognormal & 0.58 & 0.98 & 0.92 & 0.82 & 0.72 \\
\hline
\end{tabular}

The results differed depending on the probability distribution. Incidentally, the results of both cross-validation tests showed that the F-value, which is an overall index, was also the highest at 0.83 , when the distribution was an extreme value. Furthermore, both sensitivity and PPV, which are important in RSC, exceeded 0.8.

\section{DISCUSSION}

The F-values of the basic method and the proposed method with different probability distributions are shown as box plots in Figure 8. Figure 8(a) shows the results of the cross-validation for each participant, and Figure 8(b) shows the results of cross-validation for each recumbent position.

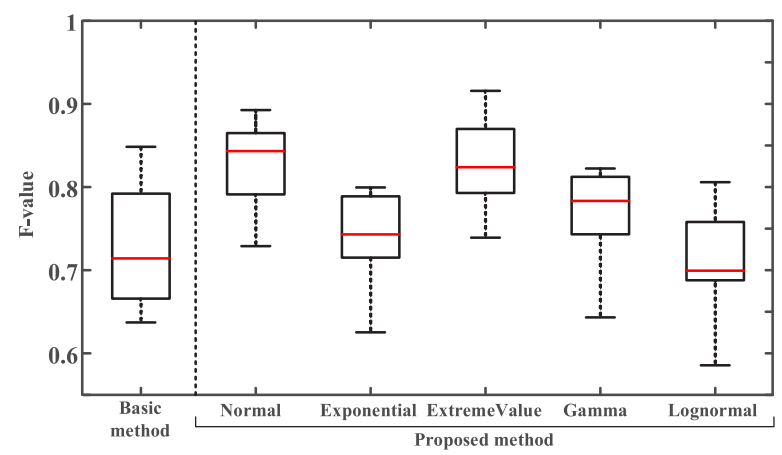

(a) Leave-one-participant-out cross-validation

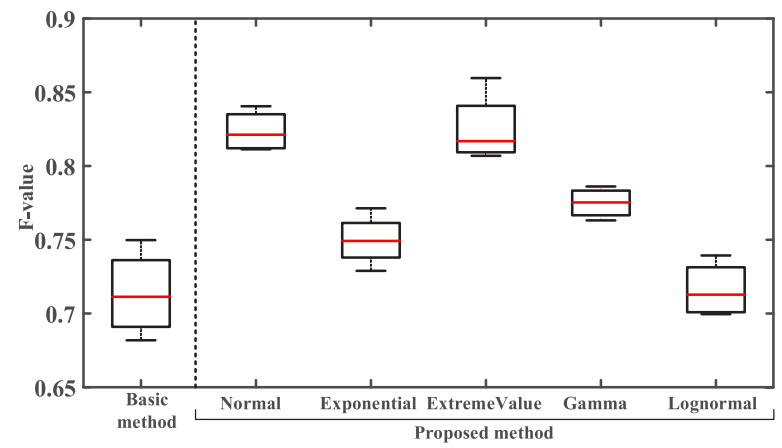

(b) Leave-one-recumbent-position-out cross-validation

Figure 8: Results of two types of cross-validation for the 10 participants 
In Figure 8(a), the median values are higher than those of the basic method for normal, exponential, extreme value, and gamma distributions, indicating that the proposed method could classify the respiratory states with higher accuracy irrespective of the differences in the characteristics of the participants.

In Figure 8(b), the median is higher than that of the basic method for all the distributions. The fact that the variability is smaller than that of the basic method also indicates that the respiratory states can be classified with higher accuracy irrespective of the variations in the physical characteristics of the participants and their recumbent positions.

These results demonstrate that the proposed method improves classification accuracy better than the basic method. Compared with the method proposed in our previous paper, the method proposed in the current paper is improved in that it can classify the respiratory states at each discrete time.

\section{CONCLUSIONS}

Respiratory frequency information fluctuates with probability, and its characteristics are believed to be different in different respiratory states. Therefore, in this study, we designed an unconstrained respiratory state classification system utilising a multidimensional probability distribution based on respiratory frequency information at each discrete time step. The performance of the proposed system was experimentally evaluated.

The results of the RSM experiment demonstrated that the respiration signals measured by the air mattress-type pressure sensor and the reference signals had an average absolute error of 0.3 , indicating high accuracy.

Furthermore, in the RSC experiment, unconstrained respiration signals were classified. The results of this validation experiment demonstrated that the proposed method outperformed the threshold method. The results of two types of cross-validation tests - conducted to determine the robustness of the proposed method to variations in the physical characteristics of the participants and their recumbent positions-demonstrated a high accuracy of F-value 0.83 when fitting the 16-dimensional extreme value distribution. The results indicate that, by considering each discrete time step, a method with a classification accuracy higher than that of the method proposed in our previous paper was obtained.

Accurate RSM and RSC were observed at each time step using the proposed system, indicating its potential application in detecting sleep diseases in a home environment.
In this study, the same type of probability distribution was applied for breathing and respiratory arrest. As there is a possibility that the optimal probability distributions for breathing and respiratory arrest are different, the verification of the proposed system with different probability distributions between breathing and respiratory arrest will be considered for future studies. In addition, five types of probability distributions were compared from perspectives other than skewness and logarithmic properties. In the future, discovering a more optimal probability distribution by comparing other probability distributions from perspectives other than skewness and logarithmic properties is being considered.

\section{REFERENCES}

1. Benjafield, A. V., Ayas, N. T., Eastwood, P. R., Heinzer, R., Ip, M.S.M., Morrell, M. J., Nunez, C. M., Patel, S. R., Penzel, T., Pépin, J. L., Peppard, P. E., Sinha, S., Tufik, S., Valentine, K., and Malhotra, A.; Estimation of the global prevalence and burden of obstructive sleep apnoea: A literature-based analysis, Lancet Respiratory Medicine, 7(8), pp.687698, 2019.

2. Nandakumar, R., Gollakota, S., and Watson, N.; Contactless sleep apnea detection on smartphones, GetMobile: Mobile Computing and Communications, 19(3), pp.22-24, 2015.

3. Gutta, S., Cheng, Q., Nguyen, H., and Benjamin, B.; Cardiorespiratory model-based data driven approach for sleep apnea detection, IEEE Journal of Biomedical and Health Informatics, 22(4), pp.1036-1045, 2018.

4. Surrel, G., Aminifar, A., Rincon, F., Murali, S., and Atienza, D.; Online obstructive sleep apnea detection on medical wearable sensors, IEEE Transactions on Biomedical Circuits and Systems, 12(4), pp.762-773, 2018.

5. Saha, S., Bhattacharjee, A., and Fattah, S. A.; Automatic detection of sleep apnea events based on inter-band energy ratio obtained from multi-band EEG signal, Healthcare Technology Letters, 6(3), pp.82-86, 2019.

6. Sadek, I., Seet, E., Biswas, J., Abdulrazak, B., and Mokhtari, M.; Nonintrusive vital signs monitoring for sleep apnea patients: A preliminary study, IEEE Access, 6, pp.2506-2514, 2018.

7. Mineharu, A., Kuwahara, N., and Morimoto, K.; A study of automatic classification of sleeping position by a pressure-sensitive sensor, Proceedings of the 2015 International Conference on Informatics, 
Electronics and Vision (ICIEV), pp.1-5, 2015.

8. Yang, C., Cheung, G., Stankovic, V., Chan, K., and Ono, N.; Sleep apnea detection via depth video and audio feature learning, IEEE Transactions on Multimedia, 19(4), pp.822-835, 2017.

9. Hwang, S. H., Lee, H. J., Yoon, H. N., Jung, D. W., Lee, Y.-J. G., Lee, Y. J., Jeong, D.-U., Park, K. S.; Unconstrained sleep apnea monitoring using polyvinylidene fluoride film-based sensor, IEEE Transactions on Biomedical Engineering, 61(7), pp.2125-2134, 2014.

10. Waltisberg, D., Amft, O., Brunner, D.P., and Tröster, G.; Detecting disordered breathing and limb movement using in-bed force sensors, IEEE Journal of Biomedical and Health Informatics, 21(4), pp.930938, 2017.

11. Zakrzewski, M., Vehkaoja, A., Joutsen, A. S., Palovuori, K. T., and Vanhala, J. J.; Noncontact respiration monitoring during sleep with microwave doppler radar, IEEE Sensors Journal, 15(10), pp.5683-5693, 2015.

12. Huang, M.-C., Xu, W., Liu, J.J., et al.; Inconspicuous on-bed respiratory rate monitoring, Proceedings of the 6th International Conference on PErvasive Technologies Related to Assistive, pp.1-8, 2013.

13. Kohama, M., Nishio, K., Hamada, Y., Kaburagi, T., and Kurihara, Y.; Unconstrained respiration states classification by detecting respiratory cycle using autocorrelation, Proceedings of the 7th International Symposium on Affective Science and Engineering, 8A-01, 2021.
Misaki KOHAMA (Non-member)

She received the B.E. degree from Aoyama Gakuin University, Japan, in 2020, where she is currently pursuing the master's degree with the Graduate School of Science and Engineering, Aoyama Gakuin University. Her research interests include bio-signal processing and system engineering.

\section{Yuri HAMADA (Member)}

She received her B.S., M.S., and Ph.D. degrees in engineering from Chuo University, Japan, in 2008, 2010, and 2017, respectively. From 2018 to 2020, she served as an Assistant Professor at Chuo University. Since 2020, she has been working as an Assistant Professor in the Industrial and Systems Engineering department at the Aoyama Gakuin University. Her research interests include modeling of communication processes and analysis of decision-making processes. She is a member of the Japan Society of Kansei Engineering, the Japanese Cognitive Science Society, and IEEE.

\section{Takashi KABURAGI (Non-member)}

He received his B.E., M.E., and Ph.D. degrees from Waseda University in 2003, 2005, and 2009, respectively. He is currently an Instructor at the College of Liberal Arts, International Christian University, where he has been a faculty member since 2019. His research interests include machine learning and time series data analysis.

\section{Yosuke KURIHARA (Non-member)}

He received his M.E. and Ph.D. degrees from Hosei University, Tokyo, in 2003 and 2009, respectively. He joined Hitachi Software Engineering, Ltd., in 2003. From 2009 to 2012, he served as an Assistant Professor at Seikei University. From 2013 to 2018, he was an Associate Professor at Aoyama Gakuin University; and from 2019, he has been serving as a Professor. He is the author of five books, more than 80 journal papers, more than 100 international conference proceedings; and holds four patents. His research interests include system engineering, sensing methods, biosensing, and system information engineering.

$\mathrm{He}$ is a member of the Japanese Society for Medical and Biological Engineering, the Society of Instrument and Control Engineers, the Electrical Engineers of Japan, the Japan Society for Fuzzy Theory and Intelligent Informatics, and the IEEE. 